\title{
Exosomes derived from human mesenchymal stem cells promote gastric cancer cell growth and migration via the activation of the Akt pathway
}

\author{
HONGBING GU $^{1 *}$, RUNBI JI ${ }^{1,2 *}$, XU ZHANG ${ }^{2}$, MEI WANG ${ }^{2}$, WEI ZHU ${ }^{2}$, \\ HUI QIAN ${ }^{2}$, YONGCHANG CHEN $^{2}$, PENGCHENG JIANG $^{3}$ and WENRONG XU ${ }^{2}$
}

\author{
${ }^{1}$ Department of Laboratory Medicine, The Affiliated People's Hospital of Jiangsu University, Zhenjiang, Jiangsu 212002; \\ ${ }^{2}$ Jiangsu Key Laboratory of Medical Science and Laboratory Medicine, School of Medicine, Jiangsu University, Zhenjiang, \\ Jiangsu 212013; ${ }^{3}$ Department of General Surgery, The Affiliated People's Hospital of Jiangsu University, \\ Zhenjiang, Jiangsu 212002, P.R. China
}

Received July 9, 2015; Accepted May 3, 2016

DOI: $10.3892 / \mathrm{mmr} .2016 .5625$

\begin{abstract}
Mesenchymal stem cells (MSCs) are a component of the tumor microenvironment and can promote the development of gastric cancer through paracrine mechanism. However, the effects of MSC-exosomes (MSC-ex) on gastric cancer are less clear. The present study reported that MSC-ex promoted the proliferative and metastatic potential of gastric cancer cells ex vivo. It was found that MSC-ex enhanced the migration and invasion of HGC-27 cells via the induction of the epithelial-mesenchymal transition. MSC-ex increased the expression of mesenchymal markers and reduced the expression of epithelial markers in gastric cancer cells. MSC-ex also enhanced the tumorigenicity of gastric cancer cells ex vivo. MSC-ex induced the stemness of gastric cancer cells. The expression of octamer-binding transcription factor 4, ex determining region Y-box 2 and Lin28B significantly increased in gastric cancer cells treated with MSC-ex. The present study further demonstrated that MSC-ex elicited these biological effects predominantly via the activation of the protein kinase B signaling pathway. Taken together, the present findings provided novel evidence for the role of MSC-ex in gastric
\end{abstract}

Correspondence to: Professor Wenrong $\mathrm{Xu}$, Jiangsu Key Laboratory of Medical Science and Laboratory Medicine, School of Medicine, Jiangsu University, 301 Xuefu Road, Zhenjiang, Jiangsu 212013, P.R. China

E-mail: icls@ujs.edu.cn

Professor Pengcheng Jiang, Department of General Surgery, The Affiliated People's Hospital of Jiangsu University, 8 Dianli Road, Zhenjiang, Jiangsu 212002, P.R. China

E-mail: pcjiang@hotmail.com

*Contributed equally

Key words: mesenchymal stem cells, exosomes, gastric cancer, epithelial-mesenchymal transition, Akt cancer and a new opportunity for improving the efficiency of gastric cancer treatment by targeting MSC-ex.

\section{Introduction}

Mesenchymal stem cells (MSCs), a heterogeneous cell population, serve important roles in cancer. They migrate to the tumor sites, differentiate into cancer-associated fibroblasts $(\mathrm{CAFs})$ and are involved in the formation of the tumor microenvironment (1). These cells exert promoting effects on tumor progression by secreting soluble cytokines, immune regulation and remodeling the tumor extracellular matrix $(2,3)$.

Exosomes, nanoscale particles secreted by numerous types of cells, deliver various signal molecules ranging from proteins, mRNAs and non-coding RNAs [microRNA (miR) and lncRNA] between cells $(4,5)$. Exosomes have been reported to be involved in reprogramming tumor behaviors, including growth, immune escape, angiogenesis, metastasis and drug resistance (6-8). Exosomes derived from breast cancer cells can transfer miR-105 targeting ZO-1 and destroy the tight junctions between endothelial cells to promote breast cancer metastasis (9). Exosomes derived from tumor stromal cells may carry mRNAs to activate the signal transducer and activator of transcription 1/NOTCH3 signaling pathway though retinoic acid-inducible gene 1 , to induce the radiation resistance in breast cancer cells (10). Therefore, exosomes are major messengers in cellular communication. However, the effects of exosomes derived from MSCs (MSC-ex) on cancer cells remain to be elucidated.

Gastric cancer has high morbidity and mortality rate worldwide (11). Revealing the causes and potential mechanisms for the development of gastric cancer is of great significance. Our previous study revealed that MSC-ex facilitated gastric cancer growth in vivo (12) and exosomes derived from gastric cancer cells stimulated CAF differentiation of MSCs (13). In the present study, the effects of MSC-ex on the malignant properties of gastric cancer cells were investigated. It was found that MSC-ex promoted the proliferative and metastatic potential of gastric cancer cells ex vivo. MSC-ex induced 
the epithelial-mesenchymal transition (EMT) and cancer stemness in gastric cancer cells. It was further demonstrated that MSC-ex activated the protein kinase B (Akt) signaling pathway in gastric cancer cells. These findings provided novel clues for understanding the role of MSC-ex in gastric cancer.

\section{Materials and methods}

Cell culture. MSCs were isolated from human umbilical cords and the experimental protocols were approved by the Ethics Committee of Jiangsu University (Jiangsu, China). Fresh umbilical cords were collected from consenting mothers and were rinsed twice in phosphate-buffered saline, containing penicillin and streptomycin. The washed cords were cut into sections of 1-3 $\mathrm{mm}^{2}$ following the removal of the cord vessels and were cultured in low glucose Dulbecco's modified Eagle's medium (DMEM; Thermo Fisher Scientific, Inc., Waltham, MA, USA) containing $10 \%$ fetal bovine serum (FBS; Thermo Fisher Scientific, Inc.), $1 \%$ penicillin and streptomycin at $37^{\circ} \mathrm{C}$ with $5 \% \mathrm{CO}_{2}$. When fibroblast-like cells reached $80 \%$ confluence, the cultures were trypsinized and passaged into new flasks for further expansion. Human fetal lung fibroblasts (HFL-1) and HGC-27 gastric cancer cells were purchased from Cell Bank of Type Culture Collection of Chinese Academy of Sciences (Shanghai, China). The HFL-1 cells were cultured in minimal essential medium- $\alpha$, supplemented with $15 \%$ FBS. HGC-27 cells were cultured in high glucose DMEM containing $10 \%$ FBS. The medium was changed every 3 days.

Isolation and characteristics of exosomes. MSCs and HFL-1 cells were cultured in serum-free medium. After $48 \mathrm{~h}$, the cell culture medium was collected and the exosomes were isolated using density gradient centrifugation, as previously described (14). Exosomes were stored at $-70^{\circ} \mathrm{C}$ until use. Exosomes derived from HFL-1 cells were used as an exosomal control. Size distribution within exosome reparations was analyzed by measuring the rate of Brownian motion using a NanoSight LM10 system equipped with a fast video capture and particle-tracking software (Nanoparticle Tracking Analysis, version 2.3; NanoSight, Amesbury, UK).

Transwell migration and invasion assays. Briefly, HGC-27 cells $\left(5 \times 10^{4}\right.$ cells $\left./ 200 \mu \mathrm{l}\right)$ suspended in serum-free medium were loaded into the upper compartment of a Transwell chamber. The lower chamber was filled with $500 \mu 1$ 10\% FBS-DMEM containing exosomes derived HFL-1 cells (HFL-ex) or MSC-ex $(80 \mu \mathrm{g} / \mathrm{l})$ in the presence or absence of LY294002 (50 $\mu \mathrm{M} / \mathrm{ml}$; Sigma-Aldrich, St. Louis, MO, USA). Following culture at $37^{\circ} \mathrm{C}$ in a humidified atmosphere of $5 \% \mathrm{CO}_{2}$ for $8 \mathrm{~h}$, the cells in the upper membrane were wiped with a wet Q-tip. The cells that had migrated through the membrane ( $8 \mu \mathrm{m}$ pore size) were fixed with $4 \%$ paraformaldehyde and stained with crystal violet. For the invasion assay, the upper compartment of a Transwell chamber was covered with $40 \mu \mathrm{l}$ matrigel and the protocol was similar to the migration assay, with the exception that the chamber was cultured at $37^{\circ} \mathrm{C}$ in a humidified atmosphere of $5 \% \mathrm{CO}_{2}$ for $24 \mathrm{~h}$. The cells were observed under a microscope and at least 6 fields of cells were assessed for each group. Each assay was repeated three times.

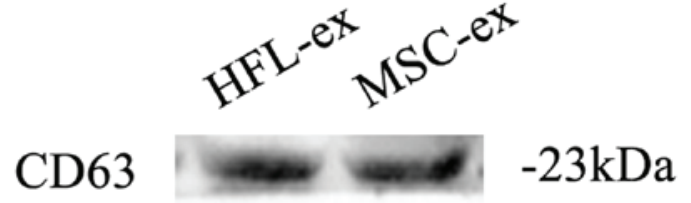

Figure 1. Western blot analysis of CD63, one of the biological markers on the surface of exosomes in the two different exosomes. MSC-ex, mesenchymal stem cell-exosomes; HFL-ex, human fetal lung fibroblast-exosomes.

Western blot analysis. The cells were collected and lysed in radioimmunoprecipitation lysis buffer, supplemented with proteinase inhibitors. Equal quantities of protein $(200 \mu \mathrm{M})$ were separated by sodium dodecyl sulfate-polyacrylamide gel electrophoresis and were subsequently transferred onto a polyvinylidene difluoride membrane. The membranes were blocked in $5 \%(\mathrm{w} / \mathrm{v})$ non-fat milk at room temperature. Following blocking, the membranes were incubated with the primary antibodies for $16 \mathrm{~h}$ at $4^{\circ} \mathrm{C}$ and secondary antibodies for $1 \mathrm{~h}$ at $37^{\circ} \mathrm{C}$. The primary antibodies were as follows: Anti-N-cadherin (1:1,000; cat. no. ab98952; Abcam, Cambridge, MA, USA), anti-E-cadherin (1:500; cat. no. sc-7870; Santa Cruz Biotechnology, Inc., Santa Cruz, CA, USA), anti-Oct4 (1:800; cat. no. 21424), anti-Sox2 (1:500; cat. no. 27015), anti-Lin28B (cat. no. 21626), anti-phosphorylated (p-)Akt (1:500; cat. no. 11501), anti-t-Akt (1:500; cat. no. 21501) (all from Signalway Antibody LLD, College Park, MD, USA), anti-Vimentin (1:1,000; cat. no. BS1491), anti-Twist (1:500; cat. no. BS60412), anti-CD63 (1:500; cat. no. BS3474) (all from Bioworld Technology, Louis Park, MN, USA) and anti-glyceraldehyde 3-phosphate dehydrogenase (GAPDH; 1:2,000; cat. no. CW0100A; Cwbio, Beijing, China). The blots were then incubated with horseradish peroxidase (HRP)-conjugated goat anti-mouse $(1: 5,000$; cat. no. CW102, 1:5,000) and goat anti-rabbit (1:5,000; cat. no. CW103) secondary antibodies (Cwbio). The signal was visualized using HRP substrate (EMD Millipore, Billerica, MA, USA) and analyzed using the LAS 4000 mini fluorescence/chemiluminescence imaging analysis system (GE Healthcare Life Sciences, Chalfont, UK).

Cell colony-forming assay. HGC-27 cells pre-treated with HFL-ex or MSC-ex $(80 \mu \mathrm{g} / \mathrm{l})$ in the presence or absence of LY294002 $(50 \mu \mathrm{M} / \mathrm{ml})$ for 7 days were collected and seeded into ultra low adhesion 6 -well plates $\left(1 \times 10^{3}\right.$ cells/well). The cells were incubated at $37^{\circ} \mathrm{C}$ in a humidified atmosphere with $5 \% \mathrm{CO}_{2}$ in serum-free medium containing growth factors, HFL-ex, MSC-ex and LY294002. The medium was changed every 3 days. The results are the mean values of three independent experiments with three replicate plates in each.

Soft agar assay. A $2 \mathrm{ml}$ agar mixture (L-DMEM + 0.6\% agar) was coated onto a 6-well plate as the bottom layer. A $2 \mathrm{ml}$ agar medium mixture (L-DMEM with $10 \% \mathrm{FBS}+0.3 \%$ agar) containing $1 \times 10^{3}$ HGC-27 cells pre-treated with HFL-ex or MSC-ex $(80 \mu \mathrm{g} / \mathrm{l})$ in the presence or absence of LY294002 $(50 \mu \mathrm{M} / \mathrm{ml})$ was added for 7 days as the top layer. 
A
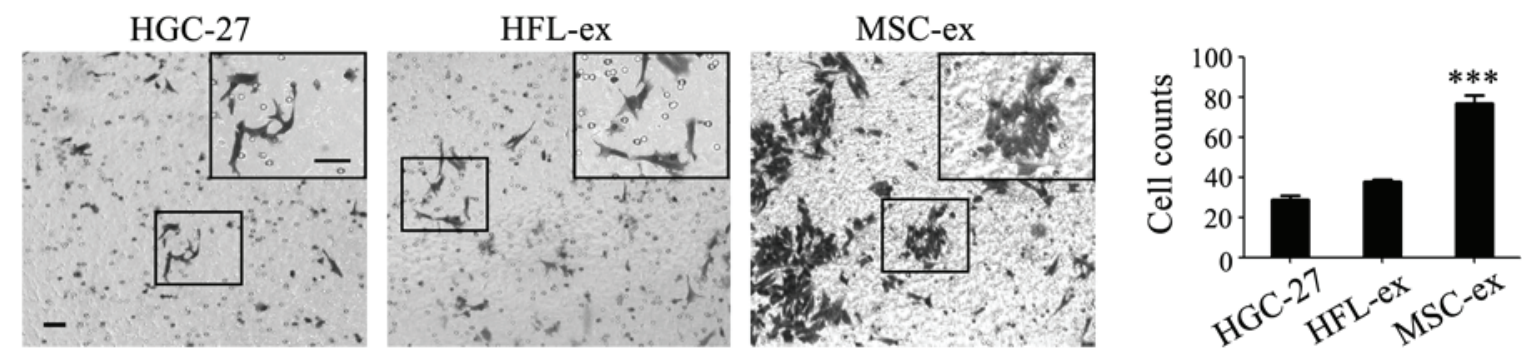

B
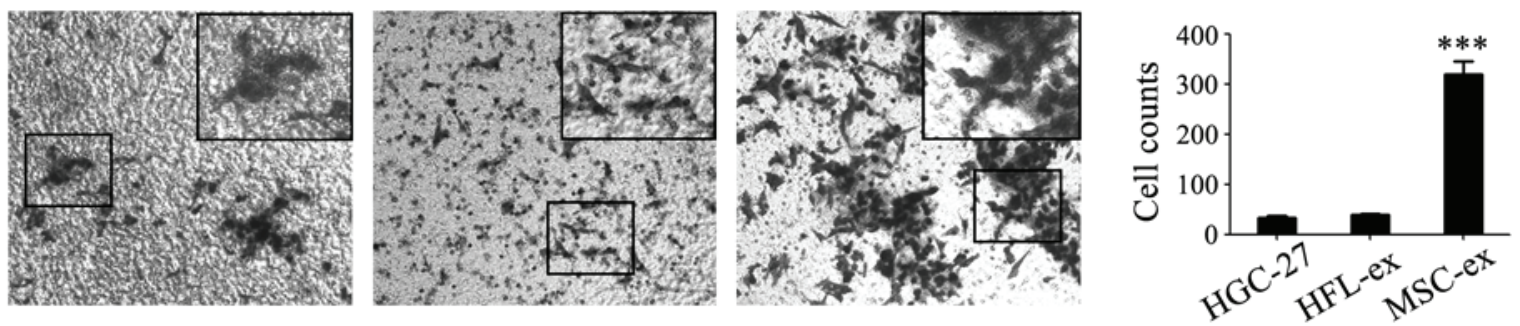

C
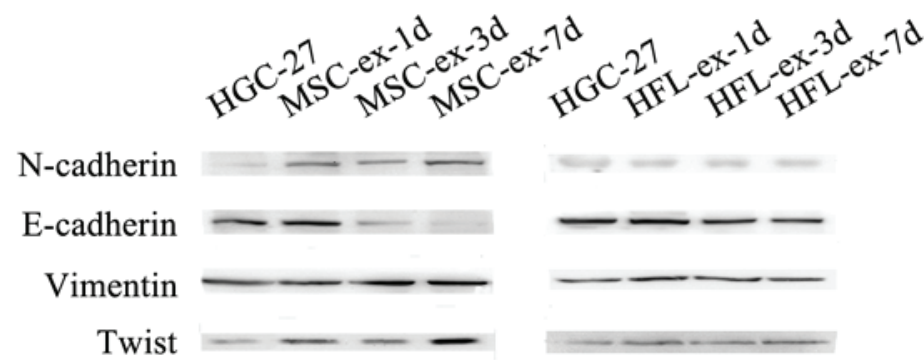

GAPDH

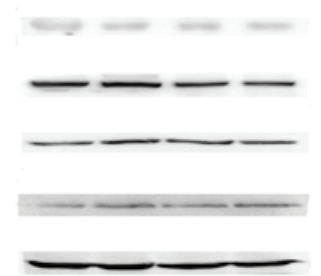

Figure 2. MSC-ex induce the EMT in gastric cancer cells. MSC-ex promoted the (A) migration and (B) invasion of HGC-27 cells. Representative images showing the migration of control untreated HGC-27 cells, HFL-ex-treated cells and MSC-ex-treated cells [magnification, x100 (large panel); magnification, x200 (small panel); scale bar, $50 \mu \mathrm{m}]$. The number of migrated cells was quantified. MSC-ex more effectively enhanced HGC-27 cells penetration through the membranes. The data are expressed as the mean \pm standard deviation $\left(\mathrm{n}=3 ;{ }^{* * * *} \mathrm{P}<0.001\right)$. (C) Western blot analysis of EMT-associated proteins, including E-cadherin, N-cadherin, Vimentin and Twist, were determined in HGC-27 cells pre-treated with MSC-ex for 1, 3 and $7 \mathrm{~d}$ and HFL-ex for 1, 3 and $7 \mathrm{~d}$. MSC-ex, mesenchymal stem cell-exosomes; HFL-ex, human fetal lung fibroblast-exosomes; EMT, epithelial-mesenchymal transition; GAPDH, glyceraldehyde 3-phosphate dehydrogenase; d, days.

After the base layer had solidified, the top layer was added. The plate was incubated for 14 days at $37^{\circ} \mathrm{C}$ in humidified atmosphere with $5 \% \mathrm{CO}_{2}$. Colony cultures were imaged and quantified.

Statistical analysis. All data are expressed as mean \pm standard deviation. SPSS software (SPSS, Inc., Chicago, IL, USA). The means of different treatment groups were compared by Student's t-test or two-way analysis of variance followed by least significant difference test. $\mathrm{P}<0.05$ was considered to indicate a statistically significant difference.

\section{Results}

Characteristics of exosomes. Exosomes derived from human MSCs and HFL-1 cells (MSC-ex and HFL-ex, respectively) exhibited a round or oval morphology, with a diameter of $79 \pm 30 \mathrm{~nm}$. as determined by using NanoSight visual nanoparticle tracking analysis. Western blot analyses revealed the positive expression of the exosomal marker CD63 in MSC-ex and HFL-ex (Fig. 1).

MSC-ex induce the EMT in gastric cancer cells. Our previous study demonstrated that tumor cells treated with
hMSC supernatant underwent EMT (15). Whether MSC-ex had similar effects on gastric cancer cells was investigated in the present study. EMT phenotypes are associated with an enhanced migration and invasion ability. Cell migration and invasion was first examined using a Transwell system. Compared with the control and HFL-ex-treated group, the MSC-ex-treatment markedly promoted the migration and invasion of HGC-27 cells (Fig. 2A and B). The number of migrating HGC-27 cells was revealed to increase by 2-3-fold (Fig. 2A) and the number of invading cells increased by 8 -fold in MSC-ex treatment group (Fig. 2B). Western blot analysis revealed that the expression of $\mathrm{N}$-cadherin, Vimentin and Twist was increased in a time-dependent manner in HGC-27 cells pre-treated with MSC-ex. By contrast, the expression of E-cadherin was notably downregulated by MSC-ex (Fig. 2C). The protein expression levels of E-cadherin, $\mathrm{N}$-cadherin, Vimentin and Twist were not significantly altered in HGC-27 cells pre-treated with HFL-ex.

MSC-ex confer stemness in gastric cancer cells. The present results showed that MSC-ex can induce the EMT in gastric cancer cells. Previous studies have reported that the EMT serves a key role in generating cancer stem cells. To explore whether 
A
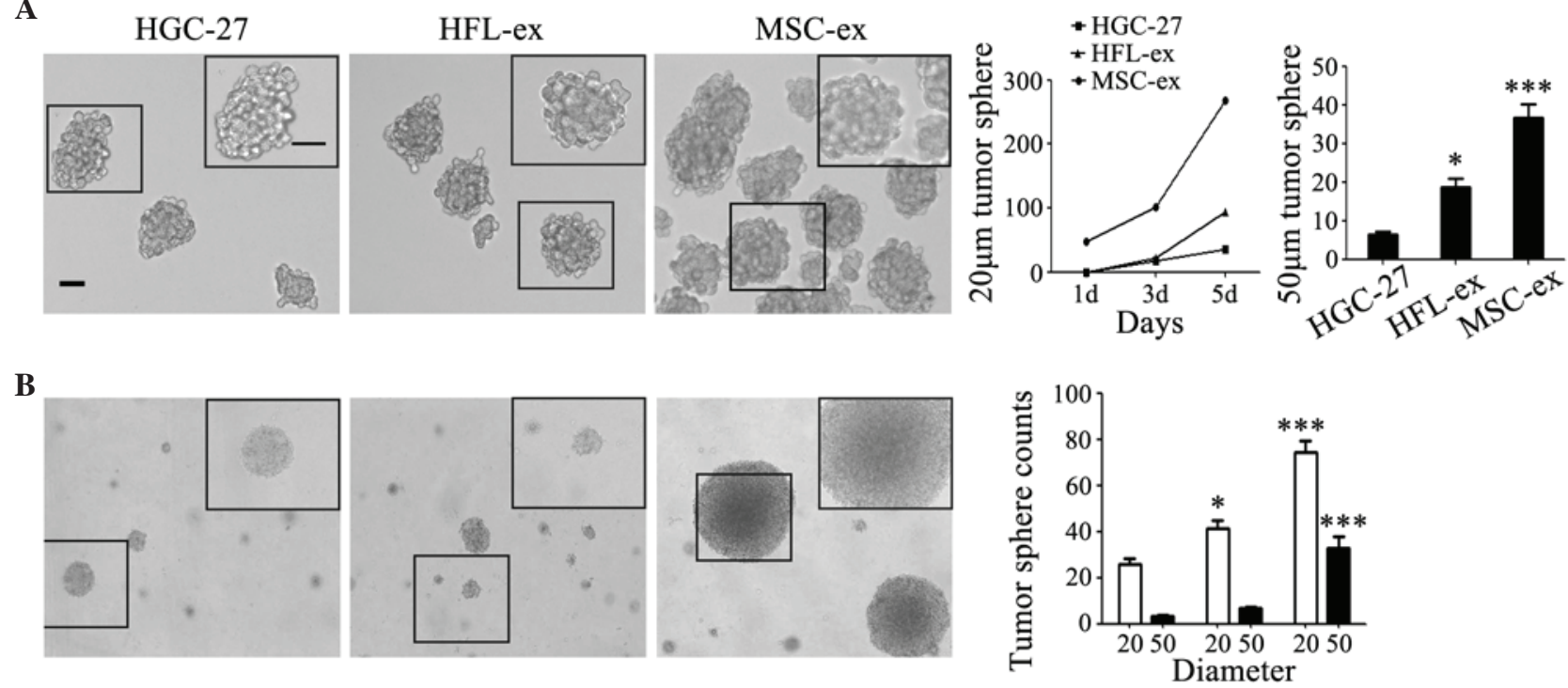

C

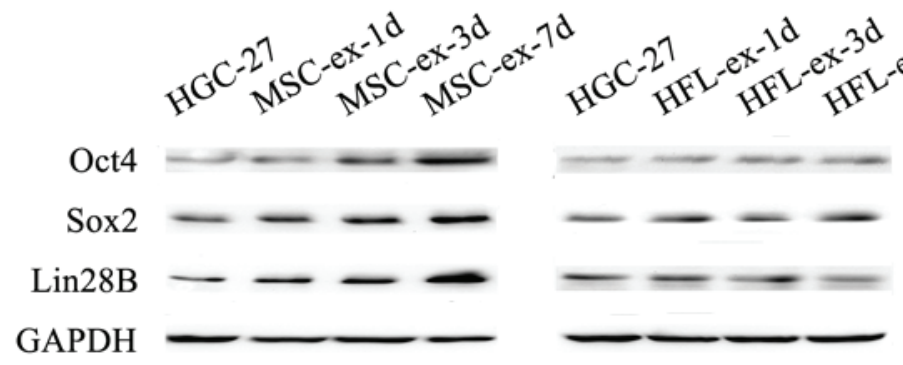

Figure 3. MSC-ex confer stemness in gastric cancer cells. (A) MSC-ex enhanced the colony formation of HGC-27 cells in serum-free medium. Representative images of cells manifesting the colonies of control untreated HGC-27 cells and cells pre-treated with either HFL-ex and MSC-ex [magnification, x100 (large panel); magnification, x200 (small panel); scale bar, $50 \mu \mathrm{m}$ ]. The formation rate of tumor spheres with a diameter of $20 \mu \mathrm{m}$ or $50 \mu \mathrm{m}$ was quantified at the fifth day. The data are expressed as the mean \pm standard deviation $\left({ }^{*} \mathrm{P}<0.05,{ }^{* * *} \mathrm{P}<0.001\right)$. (B) MSC-ex promoted the formation of HGC-27 cells spheres in semi-solid medium. Representative images of cells manifesting the colonies of control untreated HGC-27 cells and cells pre-treated with HFL-ex and MSC-ex in soft agar. Tumor spheres with a diameter of $20 \mu \mathrm{m}$ and $50 \mu \mathrm{m}$ were quantified after 2 weeks. The data are expressed as the mean \pm standard deviation ("P $<0.05$, $\left.{ }_{* * * * *} \mathrm{P}<0.001\right)$. (C) Western blot analysis of stemness-associated proteins, including Oct4, Sox 2 and Lin28B, were determined 1, 3 and $7 \mathrm{~d}$ following pre-treatment with either MSC-ex or HFL-ex. MSC-ex, mesenchymal stem cell-exosomes; HFL-ex, human fetal lung fibroblast-exosomes; GAPDH, glyceraldehyde 3-phosphate dehydrogenase; d, days; Oct, octamer-binding transcription factor 4; Sox, sex determining region Y-box 2.

MSC-ex can confer stem cell properties in gastric cancer cells, the self-renewal ability of gastric cancer cells was assessed in serum-free medium (Fig. 3A) and in semi-solid medium (Fig. 3B). HGC-27 cells in the MSC-ex treatment group formed tumor spheres with a diameter of $20 \mu \mathrm{m}$ in serum-free medium at the first day and the sphere counts reached $\sim 300$ on the fifth day (Fig. 3A). The tumor spheres with a diameter of $50 \mu \mathrm{m}$ were quantified on the fifth day, and the sphere counts in MSC-ex treatment group was $\sim 6$-fold more compared with that in the control group, and 2-fold in the HFL-ex treatment group (Fig. 3A). The results of soft agar assay revealed that MSC-ex promoted the formation of HGC-27 cell spheres in semi-solid medium. HGC-27 cells in the MSC-ex treatment group formed more and bigger colonies compared with that in control and HFL-ex treatment groups (Fig. 3B). The spheres with a diameter of $20 \mu \mathrm{m}$ in MSC-ex treatment group were $\sim 2$-3-fold more compared with that in the other groups. The spheres with a diameter of $50 \mu \mathrm{m}$ in the MSC-ex treatment group were at least 10-fold more compared with that in the control group and $\sim 5$-fold more compared with that in the HFL-ex treatment group (Fig. 3B). The expression levels of stemness-associated proteins, including Oct4, Sox 2 and Lin28B, which are known to be sufficient to reprogram somatic cells to pluripotent stem cells, were found to be notably increased in MSC-ex pre-treated HGC-27 cells compared with the HFL-ex pre-treated HGC-27 cells, as determined by western blotting (Fig. 3C). The expression of these proteins in HGC-27 cells was upregulated by MSC-ex in a time-dependent manner.

MSC-ex activate the Akt signaling pathway to promote the progression of gastric cancer cells. To determine the mechanisms by which MSC-ex promote the progression of gastric cancer cells, the present study examined the activation of Akt in MSC-ex pre-treated HGC-27 cells. As shown in Fig. 4A, the expression of p-Akt gradually increased in a time-dependent manner (Fig. 4A). Akt is one of the key downstream signaling proteins of the PI3K pathway. A specific inhibitor of PI3K, LY294002, was used to inhibit the activation of PI3K. It was found that the increased Akt phosphorylation following treatment with MSC-ex was abrogated by LY294002 (Fig. 4A). To further confirm that Akt activation is responsible for the progression of gastric cancer cells by MSC-ex treatment, HGC-27 cells were treated with MSC-ex in the presence or absence of LY294002. The increased expression of $\mathrm{N}$-cadherin, Vimentin, Twist and the decreased expression of E-cadherin were reversed by LY294002 (Fig. 4B). The enhanced 
A

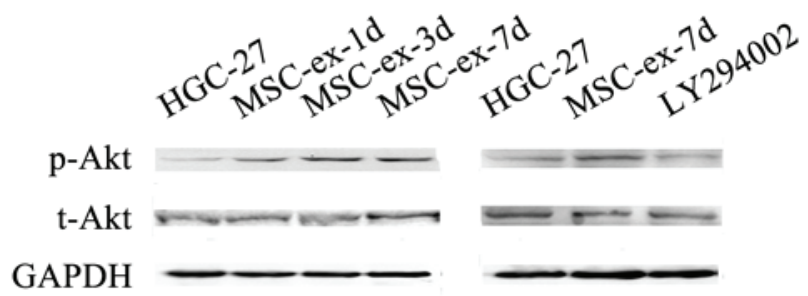

B

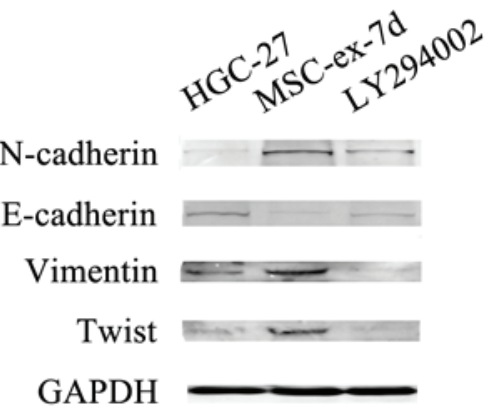

C

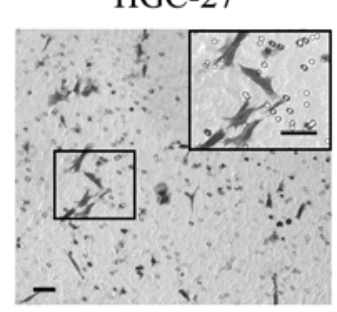

D

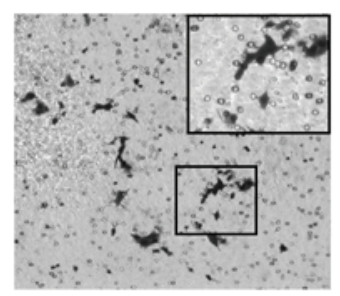

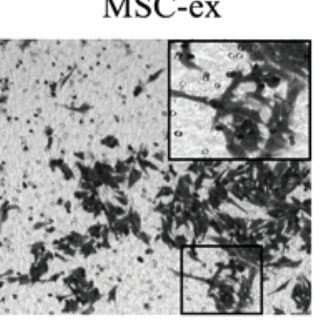

MSC-ex

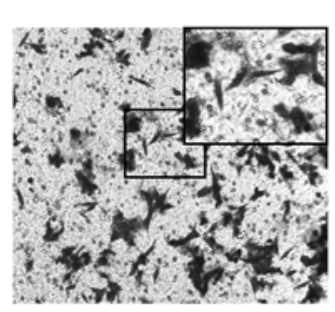

LY294002
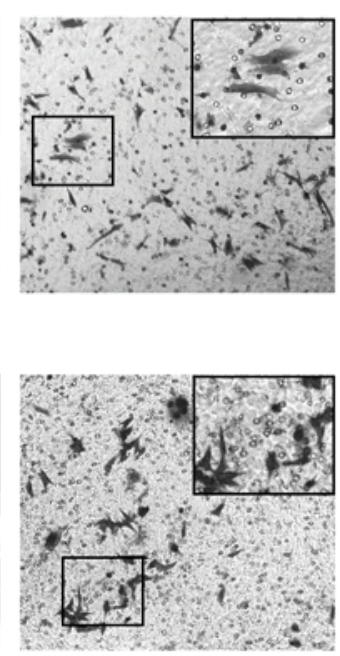
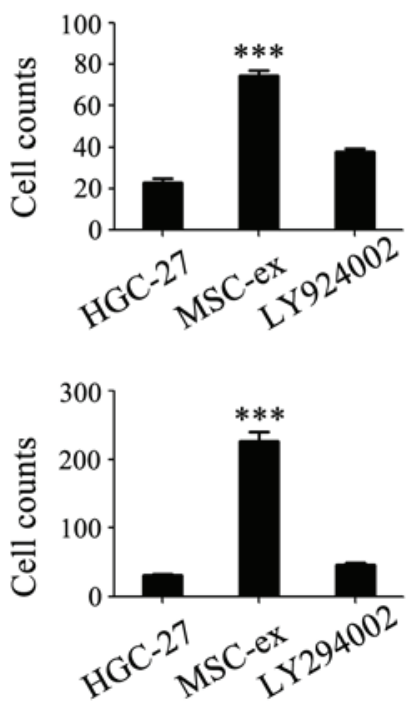

Figure 4. MSC-ex induce the EMT in gastric cancer cells via the Akt signaling pathway. (A) The expression of p-Akt gradually increased in a time-dependent manner in HGC-27 cells pre-treated with MSC-ex for 1, 3 and $7 \mathrm{~d}$, and the activation was inhibited by LY294002 as determined by western blotting. (B) LY294002 inhibited the increased expression levels of N-cadherin, Vimentin and Twist, and decreased the expression of E-cadherin in HGC-27 cells pre-treated with MSC-exosomes for $7 \mathrm{~d}$, as detemrined by western blotting. (C) LY294002 reversed the migration of HGC-27 cells enhanced by treatment with MSC-ex. Representative images demonstrated the migration of control untreated HGC-27 cells and cells pre-treated with MSC-ex in the presence or absence of LY294002 [magnification, x100 (large panel); magnification, x200 (small panel); scale bar, $50 \mu \mathrm{m}$ ]. The number of migrated cells was quantified and the data are expressed as the mean \pm standard deviation $\left.{ }^{(* * * *} \mathrm{P}<0.001\right)$. (D) LY294002 inhibited the invasion of HGC-27 cells, which was enhanced by treatment with MSC-ex. Representative images manifesting the invasion of control untreated HGC-27 cells and MSC-ex-treated HGC-27 cells in the presence or absence of LY294002 [magnification, x100 (large panel); magnification, x200 (small panel); scale bar, $50 \mu \mathrm{m}$ ]. The number of migrated cells was quantified and the data are expressed as the mean \pm standard deviation $\left.{ }^{* * *} \mathrm{P}<0.001\right)$. MSC-ex, mesenchymal stem cell-exosomes; GAPDH, glyceraldehyde 3-phosphate dehydrogenase; p-, phosphorylated; t-, total; Akt, protein kinase B; EMT, epithelial-mesenchymal transition; d, days.

migration (Fig. 4C) and invasion (Fig. 4D) of MSC-ex-treated HGC-27 cells were also inhibited by LY294002. In addition, the increased sphere counts and the sphere size caused by MSC-ex were inhibited by LY294002 both in serum-free medium (Fig. 5A) and in semi-solid medium (Fig. 5B). The formation rate of tumor spheres with a diameter of $20 \mu \mathrm{m}$ in the LY294002 group exhibited no difference with that in the control group (Fig. 5A). The number of tumor spheres with a diameter of $50 \mu \mathrm{m}$ in the LY294002 group was even lower compared with that in the control group at the fifth day after treatment (Fig. 5A). The number of formed HGC-27 cell colonies in soft agar decreased to the normal level (Fig. 5B). The induced expression levels of Oct4, Sox 2 and Lin28B proteins in HGC-27 cells were also significantly decreased by LY294002 (Fig. 5C).

\section{Discussion}

Numerous previous studies have shown that MSCs can promote the growth of different tumor types via secreting a variety of factors involved in the formation of tumor blood vessels, inducing the infiltration of inflammatory cells, or involved in the tumor microenvironment by trans-differentiation to CAF-like cells. Exosomes are important message transmitters between cells in the tumor microenvironment. They contain specific information materials from the source cells, transport proteins and nucleic acids to receptor cells, trigger downstream signaling events or change biological characteristics of the recipient cells. Our previous study demonstrated that MSC-ex accelerated the expression of $\alpha$-smooth muscle actin, vascular endothelial growth factor (VEGF), C-X-C chemokine receptor type 4 , proliferating cell nuclear antigen and increased vascular density in gastric tissues $(12,15)$. However, the effects of MSC-ex on the other aspects of gastric cancer remain unclear. Therefore, the present study investigated the effects of MSC-ex on the growth and metastatic potential of HGC-27 cells ex vivo and assessed the underlying molecular mechanism.

The present results revealed that MSC-ex enhanced the migration and invasion of HGC-27 cells ex vivo, increased 
A
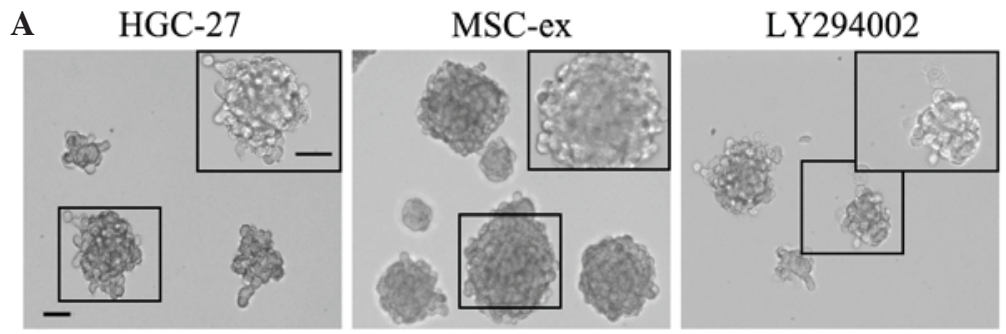

B
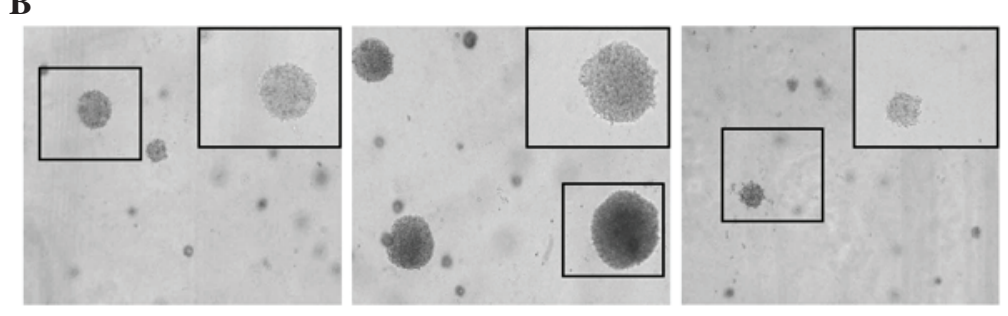
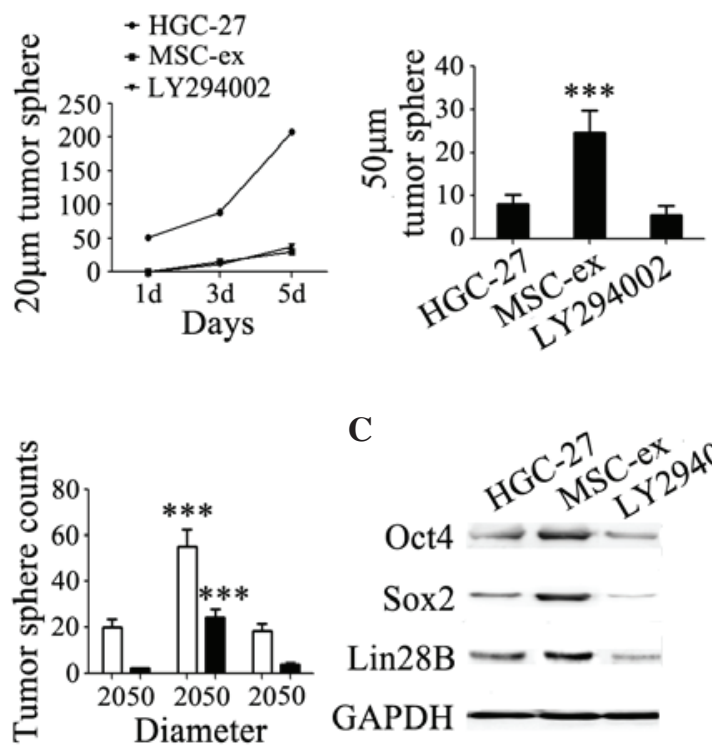

C

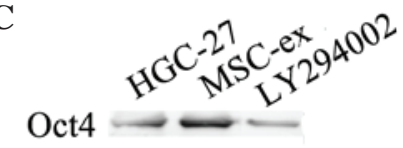

Sox 2 - -

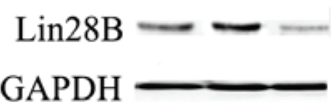

Figure 5. MSC-ex confer stemness in gastric cancer cells via the Akt signaling pathway. (A) The colony formation of HGC-27 cells in serum-free medium enhanced by MSC-ex was abrogated by LY294002. Representative images manifesting the colonies of control untreated HGC-27 cells MSC-ex-pre-treated cells for $7 \mathrm{~d}$ in the presence or absence of LY294002 [magnification, x100 (large panel); magnification, x200 (small panel); scale bar, $50 \mu$ m]. The formation rate of tumor spheres with a diameter of $20 \mu \mathrm{m}$ and $50 \mu \mathrm{m}$ ere quantified at the fifth day. The data are expressed as the mean \pm standard deviation ( $\left.{ }^{* * *} \mathrm{P}<0.001\right)$. (B) The colony formation of HGC-27 cells in semi-solid medium promoted by MSC-exosomes was abrogated by LY294002. Representative images manifesting the colonies of control untreated HGC-27 cells and MSC-ex-pre-treated cells for $7 \mathrm{~d}$ in the presence or absence of LY294002 in soft agar. The tumor spheres with a diameter of $20 \mu \mathrm{m}$ and $50 \mu \mathrm{m}$ were quantified after 2 weeks. The data are expressed as the mean \pm standard deviation $(* * * \mathrm{P}<0.001)$ (C) LY294002 inhibited the increased expression of stemness-associated proteins, Oct4, Sox 2 and Lin28B in HGC-27 cells pre-treated with MSC-ex for 7 d, as determined by western blotting. MSC-ex, mesenchymal stem cell-exosomes; HFL-ex, human fetal lung fibroblast-exosomes; GAPDH, glyceraldehyde 3-phosphate dehydrogenase; d, days; Oct, octamer-binding transcription factor 4; Sox, sex determining region Y-box 2; Akt, protein kinase B.

the expression of mesenchymal indicators and decreased the expression of epithelial indicator in HGC-27 cells. MSC-ex induced the EMT in HGC-27 cells. The EMT serves an important role in tumor invasion and metastasis. Cells experienceing the EMT are more likely to metastasize. During the EMT, tumor cells lose their epithelial cell polarity, gaining mesenchymal cell-like athleticism and can infiltrate into adjacent tissues, and this is hypothesied to be the initial stage of tumor metastasis (16). Therefore, MSC-ex promoted the occurrence of the EMT in HGC-27 cells, which may increase the metastatic capacity of gastric cancer cells.

During the procession of obtaining the EMT, the self-renewal capacity of tumor cells can also be enhanced (17). MSC-ex enhanced the tumorigenicity of HGC-27 cells in liquid and semi-solid matrix ex vivo. The sphere formation rate was higher, tumor sphere counts were increased and the tumor sphere size was bigger in MSC-ex pre-treated HGC-27 cells. Western blot analysis revealed that the expression of stemness-relevant indicators, including Oct4, Sox 2 and Lin28B, increased in HGC-27 cells pre-treated with MSC-ex compared with the control group. Therefore, MSC-ex may confer stemness in HGC-27 cells.

Akt activation is present in numerous tumor tissues, including pancreatic and breast cancer $(18,19)$. Akt is one of major downstream effectors of PI3K and activates a plurality of signal phosphorylated substrate, exerting a notable influence on cell growth and cell cycle progression. The aberrant activation of the Akt signaling pathway in malignancies can stimulate the proliferation and angiogenesis of tumor cells and is an important target for cancer therapeutics (20). In the present study, MSC-ex induced the phosphorylation of Akt, which was suppressed by using a specific inhibitor of PI3K. The inhibitor LY294002 thereby inhibited the EMT and self-renewal capacity of gastric cancer cells. Therefore, MSC-ex promoted the development of gastric cancer by activating Akt signaling pathway.

Yang et al (21) analyzed the cellular interaction between MSCs and different cancer cells by direct co-culture and found that they exchanged membrane proteins and altered functionality during bidirectional interaction. Previous studies have suggested various mechanisms elucidating the role of MSCs in the tumor microenvironment. Lee et al (22) demonstrated that MSC-ex downregulated the expression of VEGF and suppressed angiogenesis in breast cancer cells by transferring antiangiogenic molecules, including miR-16. Lin et al (23) found that MSC-ex promoted migration via the Wnt signaling pathway in breast cancer cells. MSC-ex may serve as a significant mediator of cell-to-cell communication in the tumor microenvironment. It was demonstrated that MSCs promoted migration, invasion and tumorigenicity in gastric cancer cells by exosomes instead of the physical presence of MSCs. The results of the present study provided novel evidence for the hypothesis that MSCs may function remotely through exosomes rather than in close proximity to cancer cells. The interaction between MSCs and gastric cancer cells by exosomes also imply an exosome-based therapeutic strategy. Interventions against the formation or release of exosomes, the internalization of exosomes by target cells, or the signaling cascades triggered by exosomes may improve the efficacy of gastric cancer therapy. 
In conclusion, the present findings suggested that MSC-ex induce the EMT and confer stemness in gastric cancer cells by activating the Akt signaling pathway. Exosomes may serve as a novel mediators of the promoting role of MSCs in gastric cancer and MSC-ex may represent a novel therapeutic target in gastric cancer treatment.

\section{Acknowledgements}

The present study was supported by the Major Research Plan of the National Natural Science Foundation of China (no. 91129718), the Jiangsu Province's Project of Scientific and Technological Innovation and Achievements Transformation (no. BL2012055), the Jiangsu Province for Outstanding Sci-tech Innovation Team in Colleges and Universities (no. SJK2013-10), the Jiangsu Province's Outstanding Medical Academic Leader and Sci-tech Innovation Team Program (no. LJ201117) and the Project Funded by the Priority Academic Program Development of Jiangsu Higher Education Institutions, Jiangsu Zhenjiang Science and Technology Program (no. SH2012043).

\section{References}

1. Bergfeld SA and DeClerck YA: Bone marrow-derived mesenchymal stem cells and the tumor microenvironment. Cancer Metastasis Rev 29: 249-261, 2010.

2. Cukierman E and Bassi DE: The mesenchymal tumor microenvironment: A drug-resistant niche. Cell Adh Migr 6: 285-296, 2012.

3. Barcellos-de-Souza P, Gori V, Bambi F and Chiarugi P: Tumor microenvironment: Bone marrow-mesenchymal stem cells as key players. Biochim Biophys Acta 1836: 321-335, 2013.

4. Simons M and Raposo G: Exosome-vesicular carriers for intercellular communication. Curr Opin Cell Biol 21: 575-581, 2009.

5. Pap E, Pállinger E, Pásztói M and Falus A: Highlights of a new type of intercellular communication: Microvesicle-based information transfer. Inflamm Res 58: 1-8, 2009.

6. Taverna S, Flugy A, Saieva L, Kohn EC, Santoro A, Meraviglia S, De Leo G and Alessandro R: Role of exosome released by chronic myelogenous leukemia cells in angiogenesis. Int J Cancer 130 2033-2043, 2012.

7. Hood JL, San RS and Wickline SA: Exosome released by melanoma cells prepare sentinel lymph nodes for tumor metastasis. Cancer Res 71: 3792-3801, 2011.

8. Ji R, Zhang B, Zhang X, Xue J, Yuan X, Yan Y, Wang M, Zhu W, Qian $\mathrm{H}$ and $\mathrm{Xu} \mathrm{W}$ : Exosomes derived from human mesenchymal stem cells confer drug resistance in gastric cancer. Cell Cycle 14: 2473-2483, 2015.
9. Zhou W, Fong MY, Min Y, Somlo G, Liu L, Palomares MR, Yu Y, Chow A, O'Connor ST, Chin AR, et al: Cancer-secreted miR-105 destroys vascular endothelial barriers to promote metastasis. Cancer cell 25: 501-515, 2014.

10. Boelens MC, Wu TJ, Nabet BY, Xu B, Qiu Y, Yoon T, Azzam DJ, Twyman-Saint Victor C, Wiemann BZ, Ishwaran $\mathrm{H}$, et al: Exosome transfer from stromal to breast cancer cells regulates therapy resistance pathways. Cell 159: 499-513, 2014.

11. Wang M, Gu H, Wang S, Qian H, Zhu W, Zhang L, Zhao C, Tao Y and Xu W: Circulating miR-17-5p and miR-20a: Molecular markers for gastric cancer. Mol Med Rep 5: 1514-1520, 2012.

12. Zhu W, Huang L, Li Y, Zhang X, Gu J, Yan Y, Xu X, Wang M, Qian $\mathrm{H}$ and $\mathrm{Xu} \mathrm{W}$ : Exosomes derived from human bone marrow mesenchymal stem cells promote tumor growth in vivo. Cancer Lett 315: 28-37, 2012.

13. Gu J, Qian H, Shen L, Zhang X, Zhu W, Huang L, Yan Y, Mao F, Zhao C, Shi Y and Xu W: Gastric cancer exosomes trigger differentiation of umbilical cord derived mesenchymal stem cells to carcinoma-associated fibroblasts through TGF- $\beta /$ Smad pathway. PLoS One 7: e52465, 2012.

14. Li T, Yan Y, Wang B, Qian H, Zhang X, Shen L, Wang M, Zhou Y, Zhu W, Li W and Xu W: Exosomes derived from human umbilical cord mesenchymal stem cells alleviate liver fibrosis. Stem Cells Dev 22: 845-854, 2013

15. Zhu W, Huang L, Li Y, Qian H, Shan X, Yan Y, Mao F, Wu X and Xu WR: Mesenchymal stem cell-secreted soluble signaling molecules potentiate tumor growth. Cell Cycle 10: 3198-3207, 2011.

16. Savagner P: The epithelial-mesenchymal transition (EMT) phenomenon. Ann Oncol 21 (Suppl 7): vii89-vii92, 2010.

17. Schlessinger J: Common and distinct elements in cellular signaling via EGF and FGF receptors. Science 306: 1506-1507, 2004.

18. Zhang Y, Zhang J, Xu K, Xiao Z, Sun J, Xu J, Wang J and Tang Q: PTEN/PI3K/mTOR/B7-H1 signaling pathway regulates cell progression and immuno-resistance in pancreatic cancer. Hepatogastroenterology 60: 1766-1772, 2013.

19. Gargini R, Cerliani JP, Escoll M, Antón IM and Wandosell F: Cancer stem cell-like phenotype and survival are coordinately regulated by Akt/FoxO/Bim pathway. Stem Cells 33: 646-660, 2015.

20. Davis WJ, Lehmann PZ and Li W: Nuclear PI3K signaling in cell growth and tumorigenesis. Front Cell Dev Biol 3: 24, 2015.

21. Yang Y, Otte A and Hass R: Human mesenchymal stroma/stem cells exchange membrane proteins and alter functionality during interaction with different tumor cell lines. Stem Cells Dev 24: 1205-1222, 2015.

22. Lee JK, Park SR, Jung BK, Jeon YK, Lee YS, Kim MK, Kim YG, Jang JY and Kim CW: Exosomes derived from mesenchymal stem cells suppress angiogenesis by down-regulating VEGF expression in breast cancer cells. PLoS One 8: e84256, 2013.

23. Lin R, Wang S and Zhao RC: Exosomes from human adipose-derived mesenchymal stem cells promote migration through Wnt signaling pathway in a breast cancer cell model. Mol Cell Biochem 383: 13-20, 2013. 